\title{
Research on Routing Protocols and Simulation Analysis for Opportunistic Networks
}

\author{
Wentao Wang, Feng Guo , Fang Zheng, Wan Tang and Jiangqing Wang \\ College of Computer Science, South-Central University for Nationalities, No.182 \\ Minyuan Road, Wuhan 430074, China \\ guofeng@mail.scuec.edu.cn,wangwt@mail.scuec.edu.cn
}

\begin{abstract}
Opportunistic networks are a kind of Ad Hoc networks that make use of meeting opportunities from mobile nodes to realize the network communication which doesn't need a full path between the source nodes and destination nodes. It's tolerant in latency and network division. It's a great challenge to design routing protocols in opportunistic networks in that the movements of nodes are uncertain leading to intermittent connection which make it hard to transmit message to its destination. However, the problems have also attracted more and more attention from researchers. Firstly, in this paper, we introduced the basic concept of opportunistic networks. Secondly we classified and interpreted routing protocols according to the infrastructure, scenario, forwarding mechanism etc. Thirdly, we carried out the computer simulation and analysis for opportunistic network routing protocols in aspect of message delivery ratio, network latency, routing overhead and the number of lost packets. Then we summarized the advantages and disadvantages of routing protocol and discussed its applicability. Finally we looked ahead the hotspot of recent routing protocols under opportunistic networks.
\end{abstract}

Keywords: Delay-tolerate networking, opportunistic networks, routing protocols, networks simulation

\section{Introduction}

Recently, with the increasing wireless network penetrating into our daily life, a variety of wireless terminals and applications come into our sight. How to process the division and connection interruption of wireless network appropriately is becoming more and more important. Traditional Multi-hop Wireless Networks, such as Mobile Ad Hoc Network (MANET)[1], Wireless Sensor Network (WSN)[2-4] and Deployable Ad Hoc [5], lack the specialized solution aiming at the division and connection interruption in tough environments. When fracture occurs, the network performance will degrade significantly, which causes the network unable to work normally. The demand for data communication in a challenging network environment calls for people to pay more efforts to develop the corresponding network technology. Therefore opportunistic networks [6] are proposed. Opportunistic Networks (OPN) derive from DTN [7] and MANET. It's tolerant in network latency and division. What's opportunistic networks differ from traditional Multi-hop Wireless Networks is that its nodes are not deployed with set network scale and node's initial position. Moreover, we cannot tell whether the path between the source and destination exists or not.

${ }^{*}$ Corresponding Author 


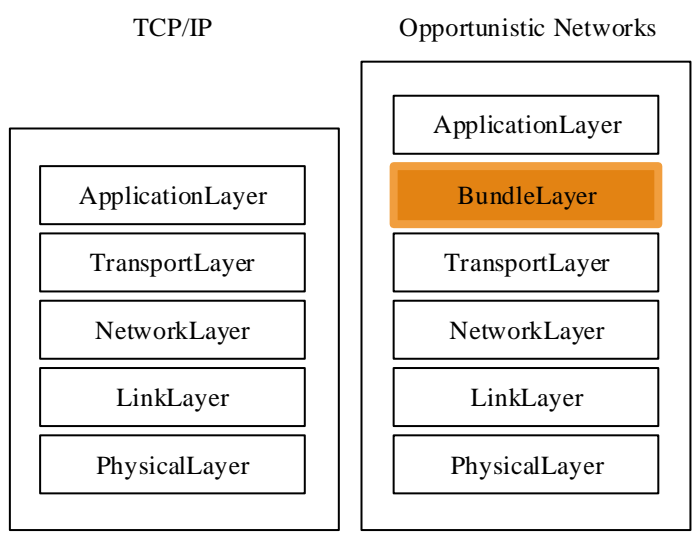

Figure 1. Protocol Stack of TCP/IP and Opportunistic Network

The architecture of opportunistic networks are different from traditional Multi-hop Wireless Networks in that they insert a new protocol layer between the application layer and transport layer to implement "storage - carry - forward" information exchange mechanism. This layer is called the bundle layer [8] shown in Figure 1. When the nodes act as hosts, bundle layer can send and receive bundle (information fused together called bundle) but cannot forward it. When the nodes serve as routers, bundle layer can store, carry and even forward the bundle as a whole. While the nodes act as gateway, the bundle of gateway requires huge storage capacity and can do security checks to make sure that the message forwarded can transmit between different network areas.

Characteristics of opportunistic network are:

(1)There isn't always an existing or complete communication path from the source to destination. What's more, the network and link would disconnect and reconnect frequently.

(2)Communication in networks are achieved by opportunistic meeting chances.

(3)Opportunistic networks are a kind of heterogeneous wireless networks including different kinds of wireless communication devices (Wi-Fi, Bluetooth, satellite etc.).

Opportunistic networks are mainly used in places where lack communication infrastructure, or even in bad network environment and emergency incidents. Opportunistic networks have the following application areas.

\section{A. Wildlife Tracking}

Zebranet [9] is a research project designed by Princeton University that uses an opportunistic networks to track wild zebra. Shared Wireless Infostation Model (SWIM) [10] uses opportunistic networks to monitor the whale's activities which installs a special Tag on whales and then collect monitor data regularly by seabirds or other floating buoys.

\section{B. Handheld Devices Networking}

The most influential one is Cambridge's Haggle's [11] project making use of handheld devices to set up networks without external access networks. They use iMote devices as network nodes to construct Pocket Switched Network (PSN). At present, multiple Haggle versions have been released under mobile devices platform such as Android, WinPhone, and IOS.

\section{Wireless Internet Access in Remote Areas}

The technology of opportunistic networks can provide non-timely, but low-cost and relatively stable network services for developing countries or remote areas where network 
infrastructures are inadequate to access the Internet. One of the internet services provided by opportunistic networks is DakNet [12]. It's deployed in India's remote areas including: Kiosk equipment in the village, MAP (Mobile Access Points) equipment on the bus, and the AP internet equipment in the town. These devices use Wi-Fi interface to communicate: Villagers exchange data by PDA and Kiosk equipment; MAP and Kiosk equipment exchange data when buses commute between rural and urban areas passing a nearby Kiosk equipment. When buses arrive at town, MAP uses AP to connect to the internet to upload or download data.

\section{Vehicle Network}

The vehicles configured with wireless intelligent devices have become the mainstream because communication between cars are urgently needed. CarTel [13] project supported by NSF (National Science Foundation) was developed by MIT based on vehicles sensor networks to collect related information. CarTel nodes can exchange data directly when cars meet. At the same time, the CarTel node can send data to the server on the internet by the roadside wireless access points.

\section{Opportunistic Network Routing Protocol}

\subsection{New Opportunistic Network Routing Mechanism}

In order to adapt to the characteristics of opportunistic networks, a new mechanism is put forward and applied to opportunistic networks including:

(1) Instead of "receive-forward", the opportunistic networks routing protocol makes use of the mechanism "receive - carry -forward" [8]. When nodes receive packets, the packets would be stored for a period of time instead of being forwarded out immediately or being dropped in a TTL time. Nodes carry the packets and then forward them out when they meet other nodes during the movement. This mechanism can overcome the problems of network division and nonexistent path from end-to-end.

(2) Nodes exchange information in pairs. Broadcast messages are difficult to reach all the nodes because of networks' division, so the pairs' exchanging mechanism has become an effective means of disseminating information. Nodes can spread information by transmitting messages that others do not have to acquire higher reliability than broadcast.

(3) Protocols have unpredictable forwarding mechanism. In some routing protocols under opportunistic networks, data packets will be forwarded many times when they meet different nodes. Multiple data copies are put into the network. This is different from traditional "one-time forward to the next finite hop".

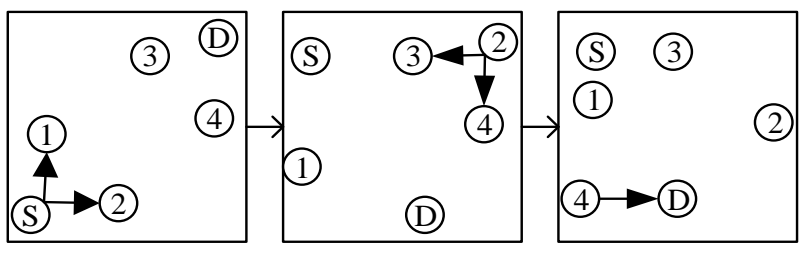

Figure 2. Illustration of Opportunistic Networks

The diagram of opportunistic network is shown in Figure 2. Source node(S) wants to transmit data to destination(D) at time $\mathrm{t} 1$, but $\mathrm{S}$ and $\mathrm{D}$ are located in different connected domains without communication path. Therefore, $\mathrm{S}$ packages data into a message and then sends it to the neighbor nodes $(1,2)$. As nodes $(1,2)$ do not have suitable opportunity to forward messages to the next-hop. They store messages locally and wait 
for a transmit opportunity. After a period of time at t2, node 2 moves to the communication range of node 3,4 . Then node 4 transmits messages to the destination D at time $\mathrm{t} 3$ to fulfill its data transmission.

\subsection{Principles and Classifications of OPN}

The design of routing protocol can be divided into two categories. One is based on the replication strategy and the other is based on the forwarding strategy [14]. The routing protocol based on replication strategy (epidemic routing) copies all packets to all nodes they meet. However simple replication strategy is a waste of resources leading to serious performance degradation. Researchers think that we can limit the number of copies and remove useless packets to improve the routing performance. Specifically:

(1) Using historical meeting information [14-16];

(2) Removing useless copies according to the information which has been transmitted;

(3) Using mobile information to predict the transmission probability;

(4) Replicating copy according to certain probability;

(5) Using network coding mechanism with redundancy;

(6) Limiting copy number of packets.

Table 1 make a simple classification according to the factors that whether routing protocols consider caching, bandwidth, as well as copying and forwarding strategies [17] or not. The protocol based on forwarding strategy only keeps one packet in the network [18]. Pelusi [6] made a routing protocol classification based on whether protocols use infrastructure or not dividing the opportunistic network routing protocol into two categories: one without the infrastructure; the other uses some forms of infrastructure, known as the infrastructure-based. Poonguzharselvi and Vetriselvi [19] classify the routing protocol into direct transmission, flooding, prediction-based, coding-based and scenario-based according to forwarding strategy.

Table 1. Classification of Some Related Work into OPN Routing Scheme

\begin{tabular}{ccccc}
\hline Problem & Storage & Bandwidth & Routing & Previous work(and mobility) \\
\hline P1 & Unlimited & Unlimited & Replication & Epidemic[21],Spray and Wait[22], \\
P2 & Unlimited & Unlimited & Forwarding & Moby Space[23](power law) \\
P3 & Finite & Unlimited & Replication & SWIM[8],Prophet[24] \\
P4 & Finite & Finite & Forwarding & Jones[25](AP TRACES) \\
P5 & Finite & Finite & Replication & MaxProp[15] \\
\hline
\end{tabular}

This paper classifies routing protocol hierarchically. Firstly, protocols are divided into infrastructure-based and non-infrastructure-based according to the infrastructure. According to different types of infrastructure, routing protocols with infrastructure can be further divided into fixed facilities and mobile devices. Station nodes in fixed facility are located in a certain position, while in mobile facilities they move in the network according to the arbitrary route. Non-infrastructure-based protocols are divided into context-based and non-context-based. Non-context-based can be further divided into direct transmission, flooding-based, utility-based and coding-based which are shown in detail in Figure 3.

Infrastructure is a particular node in the network, which have more resources and can collect information transferred by visiting nodes. 


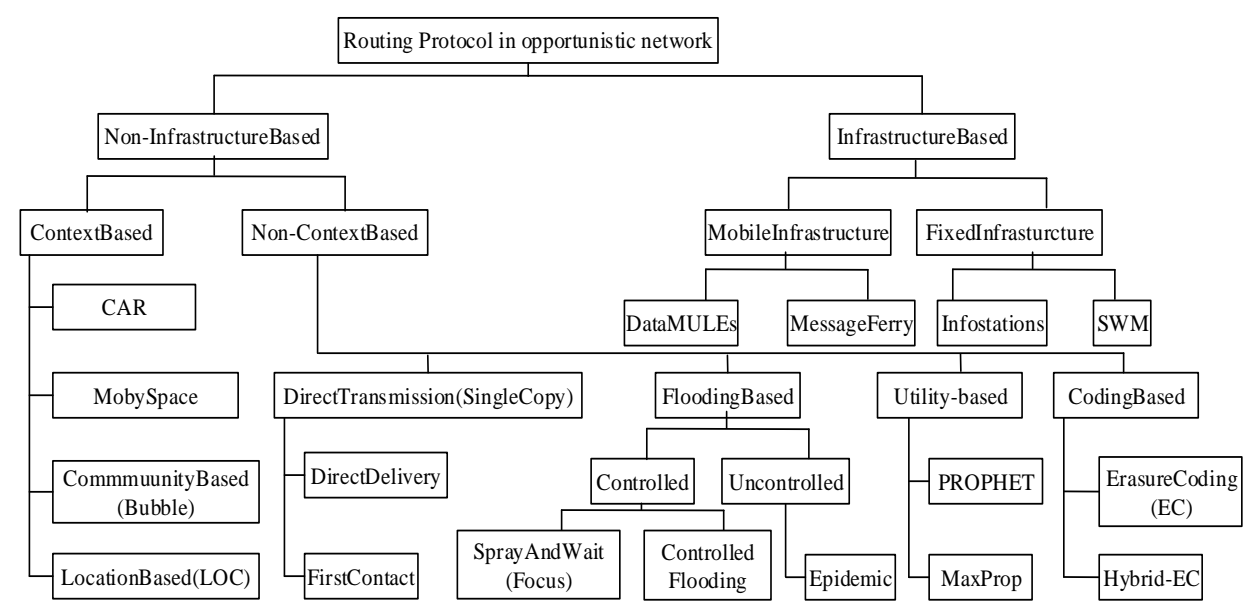

Figure 3. Classification of Opportunistic Networks Routing Protocol

\subsubsection{Routing Protocol Based on Infrastructure}

\section{A. Routing Protocol Based on Fixed Infrastructure}

Nodes in fixed facilities can be divided into stations (such as: Infostations) and normal nodes. Stations can collect data transmitted by visiting nodes. Then they transmit the data to the destination when they are within the range of communication distance.

\section{(a) Infostations}

Source nodes store messages until they reach the base station belonging to some infrastructures and then transmit messages to it [20]. The gateway of base station usually faces less challenging network (such as: they are able to provide internet access or can be connected to the LAN). Therefore, the opportunistic networks' routing algorithm is intended to convey the message to the gateway for that the gateway is easier to find the destination node [24]. However the messages experience a considerable delay.

\section{(b) SWIM (Shared Wireless Infostation Model)}

The protocol allows the connection between nodes and base stations, nodes and nodes. When a source node wants to send a message to a destination node, it can send messages to the base station directly. Otherwise, the source node passes the message to its neighbor nodes, then the data is transmitted to base station from neighbor nodes. The base station receives information and then delivers data to the destination nodes within its communication range. Jones and Ward [10] designed a system to realize the opportunistic networks to collect data in a biological information acquisition system - radio tagged whales - as nodes in a wildlife network. They derive an analytical formula for the distribution of end-to-end delays and calculate the storage requirements. They further extend SWIM by allowing multi-tiered operation, which in their biological information acquisition system could be realized through seabirds acting as mobile data collection nodes.

\section{B. Routing Protocol Based on Mobile Devices}

Mobile devices move in mobile-based network according to arbitrary or special designed route. They serve as data collectors collecting data from nodes to realize transmission [25]. 


\section{(a) MessageFerry}

Reference [26] pointed out that Ferry nodes are used to provide forwarding services. They collect information from the source during the movement in network. They assumed that only a single ferry is present in the system and this ferry never fails. There are two ferry route schemes. The first scheme referred to the earliest deadline (ED) scheme. Ferry maintains a list of nodes that need to be visited either because messages needed to be picked up or dropped off. This list is ordered based on the deadlines of the messages (assuming that the reservation message indicates the message deadlines). After the ferry has visited the current node, it picks the node with the earliest deadline to visit next. The second scheme is called Elliptical Zone Forwarding (EZF) scheme. The ferry maintains a list of destinations that need to be visited and another list of reservations that it has received (i.e., nodes with messages that need to be picked up). After the ferry has visited a node, it checks its destination list and performs the following computation to decide if there is any node in the reservation list that it can visit before visiting a node in the destination list with the earliest deadline. Otherwise, the ferry will just visit the next node in the destination list with the earliest deadline. Such an approach is taken in an attempt to reduce the overall message delay while maintaining a high delivery ratio for urgent messages. Note that we assume that all packets that are delivered can meet the deadline requirements.

\section{(b) DataMULEs (Data Mobile Ubiquitous LAN Extensions)}

Reference [27] pointed that DataMULEs is mainly used in sparse wireless sensor network to collect data. It's composed of three layer: The bottom layer uses sensor nodes to collect data from the surrounding environment periodically. The middle layer is composed of mobile nodes. They move around the field of the sensor collecting data and transmitting information to station. The top layer is composed of a set of cable Aps and data storage. They receive the data from the mobile node and then send the data to station for storing and following process.

\subsubsection{Routing Protocol Based on Particular Scenario}

\section{A. CAR (Context-Aware Routing)}

This protocol is proposed by Musolesi [28] et al., Each node in this protocol is responsible for calculating transfer probability. The probability is that they can reach their destination node and then exchange transfer probability periodically. Each node calculates which one is the most suitable intermediate node to achieve the transmission.

Transfer probability can be calculated through a set of context information related to a certain host which can be defined using a set of attributes (X1, X2, Xn). Those attributes denoted with a capital letter (e.g., X1) refer to the set of all possible values for the attribute, whereas those denoted with a lower case letter (e.g., x1) refer to a particular value within this set. In the remainder of this section we will use the classical notation of utility theory. Our goal is to allow each host locally to associate a utility function $\mathrm{U}(\mathrm{x} 1$, $\mathrm{x} 2, \ldots, \mathrm{xn})$, representing the delivery probability with every other host.

Our aim is to maximize each attribute and choose the host that presents the best tradeoff between attributes and system message delivery ratio. The combined goal function used in the weight method can be defined as:

$$
\text { Maximise }\left\{f\left(U\left(x_{i}\right)\right)=\sum_{i=1}^{n} a_{i}\left(x_{i}\right) w_{i} U_{i}\left(x_{i}\right)\right\}
$$

Reference [28] show that if the node's cache is small, the CAR of the routing protocol packet transmission rate is higher than Epidemic routing protocol because each message 
in the CAR routing protocol only produce a copy, saving storage space and network bandwidth resources. While node's cache becomes larger, the result is opposite.

\section{B. MobySpace}

This protocol is proposed by Leguay [28] et al, which sets up high dimensional Euclidean space [10], named MobySpace. Each axis of the space represents the possibility of connection in a pair of nodes, and the length of axis indicates the possibility of contacting. The best forwarding node is the one that is closest to the destination node. Obviously we need to know information of all the axis in the virtual space, and also all the nodes' information. Compared with the Epidemic routing protocol, MobySpace routing protocol consumes less in the consumption of storage and network bandwidth resources.

\subsubsection{Non-specified Scenario Routing Protocol}

\section{A. Single Copy simple routing protocol}

\section{(a) FirstContract}

FirstContact [29] routing protocol transmits message when nodes meet. Through multiple relay, messages are eventually passed to the target or discarded due to timeout. Data packet is not copied by nodes during transmission. There is only one copy in the entire network.

\section{(b) DirectDelivery}

DirectDelivery [30] only sends data packet to the destination when they meet, but don't exchange messages if the next-hop isn't the target. Data packet is not copied by nodes during transmission. Therefore, there is also only one copy in the whole network.

\section{B. Multi-copy flooding routing protocol}

\section{(a) Epidemic}

Amin and David proposed the epidemic routing protocol [21]. The protocol relies upon the transitive distribution of messages through opportunistic networks with messages eventually reaching their destination. Each Node maintains a buffer consisting of messages that it has originated as well as messages buffered on behalf of other nodes. For efficiency, a hash table indexes this list of messages, keyed by a unique identifier associated with each message. Each node stores a bit vector called the summary vector. The vector indicates which entry is set in local hash tables. When two nodes come into communication range of one another, the node with the smaller identifier initiates an antientropy session with the larger identifier node. To avoid redundant connections, each node maintains a cache of nodes that it has spoken with recently. Two nodes exchange their summary vectors to determine which messages stored remotely have not been seen by the local node. In return, each node then requests copies of messages that it has not yet seen. The receiving node maintains total autonomy in deciding whether it will accept a message. For example, it may determine that it is unwilling to carry messages larger than a given size or destined for certain nodes. While we do not experiment with such general policies, we do model a maximum queue size associated with each node, which determines the maximum number of messages of a node that is willing to carry on behalf of other nodes.

When node A comes into contact with node $B$. In step one, A transmits it summary vector $\mathrm{SV}_{\mathrm{A}}$ to $\mathrm{B} . \mathrm{SV}_{\mathrm{A}}$ is a compact representation of all the messages being buffered at $\mathrm{A}$. Next, B performs a logical AND operation between the negation of its summary vector: $S V_{B}$ (the negation of B's summary vector, representing the messages that it 
needs) and $S V_{A}$ as in (2). In step three, A transmits the requested messages to B. This process is repeated transitively when $\mathrm{B}$ comes into contact with a new neighbor. Given sufficient buffer space and time, these sessions guarantee eventual message delivery through such pair-wise message exchange.

request $S V_{B}=S V_{A}-S V_{A} \cap S V_{B}$

\section{(b) Controlled Flooding}

\section{Controlled Flooding}

Controlled Flooding proposed by Khaled et al., [31] in accordance with the operational details can be divided into BP (Basic Probabilistic) algorithm, BP + TTL (Time to Live), BP + Kill Time and BP + Passive Cure.

(1) Basic Probabilistic (BP): To more closely emulate reality, they choose a uniform distribution probabilistic function that determines the willingness of the nodes to transmit a given message. When talking about the willingness of a node, they assumed that forwarder nodes have the same willingness as the sender. Based on the result of this function, a forwarder may choose not to forward the message at all, forward it at half the willingness of the sender or forward it at the same level of willingness as the sender.

(2) Time-to-Live (TTL): In this scheme, they add a time-to-live value (TTL >=1) as the number threshold of message forwarding on the basis of BP controlled flooding algorithm. The setting of TTL threshold helps to reduce flood operational overhead, but its suitable value is difficult to determine. The TTL here determines how many times the message is forwarded before it is discarded. They add the TTL on top of the BP scheme since the BP scheme is a more realistic representation of how nodes act regarding the choice of forwarding messages.

(3) Kill Time: The time stamp is the time interval after which the message should no longer be forwarded. Here, they add a time stamp to the message on top of the BP scheme. This could be very useful if the sender node knows how long it will be disconnected. This is also a good way to set the maximum time that a node should keep a message in its buffer if the times-to-send (TTS) variable of that message does not reach zero.

(4) Passive Cure: It's a final scheme or optimization. The idea is that once the destination (Ultimate node) receives the message, it generates a Passive Cure to "heal" the nodes in the network after they have been "infected" by the message.

\section{Spray and Wait}

Spray and Wait proposed by Spyropoulos et al., [22] is based on flooding strategy to limit the messages overhead. They aim at: perform significantly fewer transmissions than epidemic and other flooding-based routing schemes under all conditions; generate low contention, especially under high traffic loads; achieve a delivery delay that is better than existing single and multi-copy schemes, and close to the optimal; be highly scalable; be simple and require as little knowledge about the network as possible, in order to facilitate implementation. Spray and Wait routing consists of the following two phases:

1) Spray phase: for every message originating at a source node, $L$ message copies are initially spread-forwarded by the source and possibly other nodes.

2) Wait phase: if the destination is not found in the spraying phase, each of the nodes carrying a message copy performs direct transmission (i.e., will forward the message only to its destination).

Spray and Wait routing protocol can reduce the flooding overhead, but the selection of parameter $\mathrm{L}$ value is difficult. If $\mathrm{L}$ is too high, cost will be increased; if not, the delivery ratio will decrease. Spyropoulos [21] gives the relationship between latency and L. L is 
the expected delay of Spray and Wait. When message copies are used, L is upperbounded by

$E D_{S W} \leq\left(H_{M-1}-H_{M-L}\right) E D_{d t}+\frac{M-L}{M-1} \frac{E D_{d t}}{L}$

His bound is tight when $\mathrm{L}<\mathrm{M}$. The equation (3) can be further simplified to

$\left(H_{M}^{3}-1.2\right) L^{3}+\left(H_{M}^{2}-\frac{\pi^{2}}{6}\right) L^{2}+\left(\mathrm{a}+\frac{2 M-1}{M(M-1)}\right) L=\frac{M}{M-1}$

$H_{\mathrm{n}}^{r}=\sum_{i=1}^{n} \frac{1}{i^{r}}=\theta(\log n)$

Where $H^{r}$ is the nth Harmonic number of order $\mathrm{r}$.

Binary Spray and Wait [21] is an improved protocol based on Spray and Wait which performs better. In this protocol, the source node send half of copies to new intermediate nodes, leaving another half of the rest for itself ( $\mathrm{L}=\mathrm{n} / 2$ compared to Spray and Wait). Then the source node and relay node repeat the process, until only one copy left and it goes into Wait parse directing transmitting to the destination node. The fastest way is binary spray. Since replication distribution uses a binary tree, it can distribute the packet out in parallel at a faster speed. So Binary Spray and Wait is better than Spray and Wait on the delivery rate and delay.

Existing spraying schemes generate and distribute ("spray") a small, fixed number of copies or "forwarding tokens" to a number of distinct relays. This problem could be solved if a sophisticated single copy scheme is used to further route a copy after it's handed over to a relay. A scheme that takes advantage of transmissions (unlike Direct Transmission) is another improvement of (Binary) Spray and Wait. With this in mind, Spyropoulos [32] proposes Spray and Focus, which in the second phase ("focus" phase) rather than waiting for the destination to be encountered, each relay can forward its copy to a potentially more appropriate relay, using a carefully designed utility-based scheme. Spraying out the messages in a short time will do well to the delivery latency.

Figure 4 compared the forwarding similarities and differences of SF (Spray and Focus) and SW (Spray and Wait). SW uses Direct-Delivery way (mainly in the second phase) waiting for the destination. SF uses relay based on utility which messages forward along a high utility node (gray) until they meet the target. When utility distribution is reasonable, SF could improve the success rate of delivery greatly.
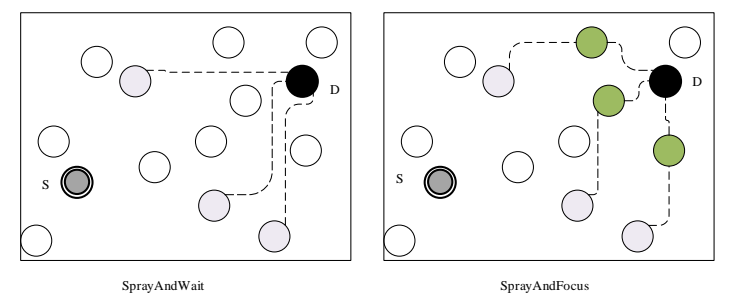

\section{Figure 4. Last Copy Transmitted by SW and SF}

\section{Utility Based}

\section{(a) Prophet}

Prophet (Probabilistic Routing Protocol using the History of Encounters and Transitivity) proposed by Lindgren, et al [24] is based on probability. Most nodes usually do not move around completely randomly and movement patterns are thus likely to be predictable. If a location has been frequently visited in the past, it is likely that it will be 
visited again in the future. We would like to make use of this observation to improve routing performance by doing probabilistic routing. A probabilistic metric called delivery predictability is established at each node. They could know the possibility indicating the predicted chance of that node delivering a message to that destination. When a node encounters another, they exchange information about the delivery predictabilities they have and update their own information accordingly. Based on the delivery predictabilities, a decision is then made on whether or not to forward a certain message to this node.

The protocol relies on the delivery predictability metric as in (6). $P \in[0,1]$. That should reflect the probability of encountering a certain node. The metric should be used to support the decision of whether or not to forward a message to a certain node. Whenever a node is encountered, the metric should be updated according to (6), where $\mathrm{P}(\mathrm{a}, \mathrm{b})$ is the delivery predictability node a has for node $\mathrm{b}$, and $P_{\text {init }} \in[0,1]$ is an initialization constant. This ensures that nodes that are often encountered have a high delivery predictability.

$P_{(a, b)}=P_{(a, b) \text { old }}+\left(1-P_{(a, b) \text { old }}\right) \times P_{\text {init }}$

If a pair of nodes do not encounter each other in a while, then the transmission probability will gradually degenerate. They are less likely to be good forwarders of messages to each other. Thus the delivery predictability values must age, being reduced in the process. The aging equation is in (7), where $\gamma \in[0,1]$ is the aging constant, and $\mathrm{k}$ is the number of time units that have elapsed since the last time the metric was aged. The time unit used can differ, and should be defined based on the application and the expected delays in the targeted network.

$P_{(a, b)}=P_{(a, b) o l d} \times \gamma^{\kappa}$

The delivery predictability also has a transitive property, that is based on the observation that if node A frequently encounters node B, and node B frequently encounters node $\mathrm{C}$, then node $\mathrm{C}$ probably is a good node to forward messages destined for node A to. Equation (8) shows how his transitivity affects the delivery predictability, where $\beta \in[0,1]$ is a scaling constant that decides how large impact the transitivity should have on the delivery predictability.

$P_{(a, c)}=P_{(a, c) \text { old }}+\left(1-P_{(a, c) o l d}\right) \times P(a, b) \times P(b, c) \times \beta$

\section{(b) MaxProp}

Burgess et al., [15] proposed the MaxProp. They assumed that each peer has an effectively unlimited buffer for messages that they originate, but a fixed-size buffer for carrying messages originated by others. They assume that transfer opportunities are limited both in duration and bandwidth. They assume peers have no a priori knowledge of network connectivity, no control over their movement, no knowledge of geographic location, and there are no always-on stationary peers in the environment. MaxProp cares for three stages:

1) Neighbor Discovery. Peers must discover one another before a transfer opportunity can begin; they do not know when the next opportunity will begin. A more efficient fast Neighbor Discovery is put forward in [35].

2) Data Transfer. When two peers meet, the amount of data they can transfer is limited. Peers do not know the duration of each opportunity.

3) Storage management. As packets are received from a neighbor, each peer must manage its finite local buffer space by selecting packets to delete according to some algorithm. Messages that are destined for a receiving peer are passed up to the application layer and removed from the buffer. 
Estimating Delivery Likelihood: Previous work has demonstrated that optimal delivery paths in opportunistic networks can be discovered by constructing a directed graph of nodes connected by edges representing traversals through time and space. A variation of Dijkstra's algorithm can determine the shortest path if one exists. In practice, no oracle is available to reveal future connections. MaxProp therefore assigns link weights as follows.

Let the set of nodes in the network be $\mathrm{s}$. Each node, $\mathrm{i} \in s$, keeps track of a probability of meeting peer $\mathrm{j} \in s$. They estimate this probability $\mathrm{f}_{j}^{i}$ as the likelihood that the identity of the node they connect to next will be $\mathrm{j}$. For all nodes, $\mathrm{f}_{j}^{i}$ is initially set to $\frac{1}{|s|-1}$. When node $\mathrm{j}$ is encountered, the value of $\mathrm{f}_{j}^{i}$ is incremented by $1\left(\mathrm{f}_{j}^{i}=\mathrm{f}_{j}^{i}+1\right)$, and then all values of $f$ are re-normalized. Using this method, often called incremental averaging, nodes that are seen infrequently obtain lower values over time. In MaxProp, each time two peers meet, they exchange these values with one another.

For example, for an opportunistic network with four other nodes, a peer $\mathrm{j}$ has values for $f_{1}^{i}=f_{2}^{i}=f_{3}^{i}=f_{4}^{i}=0.25$. Upon encountering node 3 , the peer sets $f_{3}^{i}=1.25\left(f_{3}^{i}=f_{3}^{i}+1\right)$ and renormalizes all values so that they sum to 1 again $\left(\sum \mathrm{f}_{j}^{i}=1\right): \mathrm{f}_{1}^{i}=\mathrm{f}_{2}^{i}=\mathrm{f}_{3}^{i}=\mathrm{f}_{4}^{i}=0.125$ and $\mathrm{f}_{3}^{i}=0.625$. With other nodes' values in hand, a local node calculates a cost $\mathrm{c}(\mathrm{i}, \mathrm{i}+1 \ldots \mathrm{d})$ for each path possible to the destination $\mathrm{d}$ up to $\mathrm{n}$ hops long. The cost for a path using nodes $i, i+1$, d. Cost is the sum of the probabilities that each connection on the path does not occur, estimated as one minus the probability that each link does occur:

$\mathrm{c}(\mathrm{i}, \mathrm{i}+1, \ldots, \mathrm{d})=\sum_{\mathrm{k}=i}^{d}\left(1-f_{i+1}^{i}\right)$

The cost for a destination is the lowest path cost among all possible paths. Figure 5 illustrates an example of this policy where the cost from A to D is determined as the minimum value 1.25. In practice, this calculation among all possible paths is fast because paths monotonically increase in cost during a depth-first search. Once the cost for a path is worse than the current best path, the search can stop. In our evaluations, they set the maximum path length to search as 10 . Packets that are ranked with highest priority are the first to be transmitted during a transfer opportunity. Packets ranked with lowest priority are the first to be deleted to make room for an incoming packet. When two packets have destinations with the same cost, the tie broken by giving the packet that has traveled fewer hops higher priority.

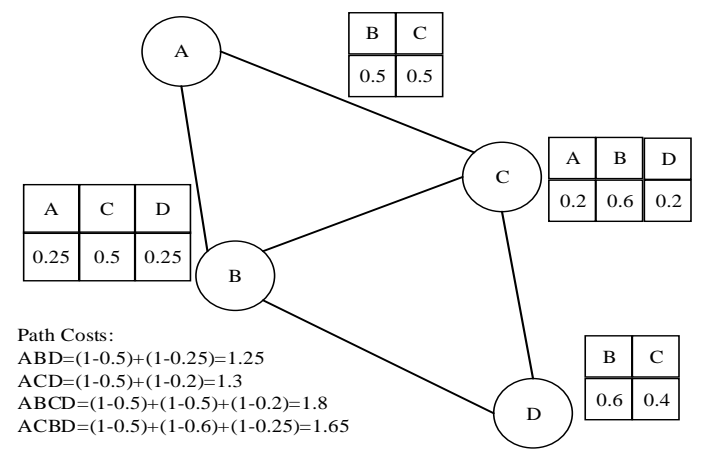

Figure 5. The MaxProp Routing Strategy 


\section{Coding Based}

\section{(a) EC (Erasure Coding)}

We will give a brief overview on erasure code and a forwarding scheme proposed by Wang et al., in [34]. Erasure coding is a coding scheme which provides better faulttolerance by adding additional information (such as redundancy [33] or decoding [34]) without the overhead of strict replication to the original data.

Erasure codes operate by converting a message into a larger set of code blocks such that any sufficiently large subset of the generated code blocks can be used to reconstruct the original message. The algorithm produces $M * r / b$ equal sized code blocks of size $\mathrm{b}$, such that any $(1+\varepsilon) \cdot M / b$ erasure coded blocks can be used to reconstruct the message. Here, $\varepsilon$ is a small constant and varies depending on the exact algorithm used, such as Reed-Solomon codes or Tornado codes. Erasure Coding based forwarding algorithm can be understood as an enhancement to the simple replication algorithm.

Generally speaking, supposing that a message has $\mathrm{M}$ bytes and replicator is $\mathrm{r}$. After coding, the message can be divided into several data partition which has b bytes. Block number $N=\frac{M \times \mathrm{r}}{b}$, when receiving $\frac{N}{\mathrm{r}}$ packet can restructure the original message. The following Figure 6 (we call it "Two-Hop" scenario[36]), the coding block evenly distribute to $\mathrm{n}$ relay nodes, and each relay node can receive the number of coding block as shown in (10):

$\frac{N}{n}=\frac{M r}{b n}$

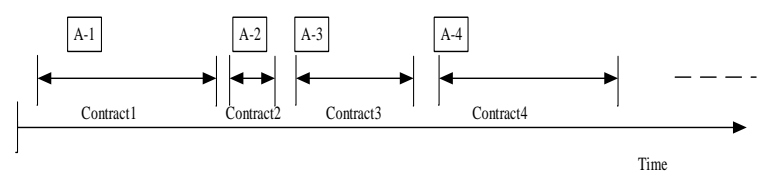

\section{Figure 6. Illustration of the EC. Each Coded Block is Equally Split into 4} Sub-blocks $(n=4)$

A-EC (aggressive erasure coding) [37] overcomes the drawback of EC. What's different from EC is that it transfers as much as possible the encoding block during each contact time.

Widmer [33] proposed an opportunity forward mechanism based on random linear network coding. The schema map the different sources of information to a limited domain to form an information vector. While receiving messages with the specific number, the protocol linearly encodes these messages into message vector and injects into the network. When the destination node receives enough messages vector it can decode the original message. Forward mechanism based on coding has good robustness in network congestion or packet loss due to bad link signal. It can also control network overhead perfectly and has a good expandability.

\section{(b) H-EC}

H-EC proposed by Ling et al [38] which is a kind of hybrid routing mechanism makes full use of robustness of the EC routing protocol. It also retains characteristics of the copy forwarding mechanism. Source nodes in this protocol generate parts of the encoded messaged into two copies. When they encounter the middle node, they adopt the way of A-EC to send more fragmentations as more as possible. As shown in Figure 7, the difference is that the first copy passed to it which is similar to the EC routing protocol. 
And then during the rest of the connection time, they pass the second copy to the node making full use of every connection opportunity to get better forwarding performance.

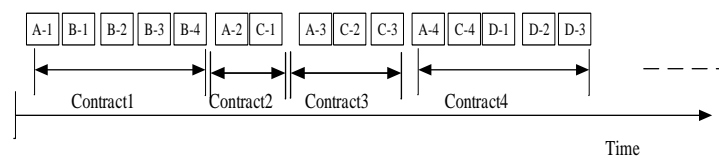

\section{Figure 7. Illustration of the H-EC schema. Each Coded Block is Equally Split into 4 Sub-blocks $(n=4)$}

\subsection{Comparison among Different Routing Protocols}

Through the comparison, we can conclude that each protocol has its own advantages and disadvantages. Routing protocol with storage managing could save routing overhead, but it may affect the success delivery rate of the transmission. So we must carefully consider its management strategy. Because of the physical limit of cache, cache management strategies are needed in the actual situation. How to reduce the influence of cache management strategy on network performance is worth further researching. Routing protocol that can predict the probability of nodes' meet is able to reduce useless message and routing overhead. But it affects the transfer rate of packets because probability estimation based on historical information might be different with the actual situation. But exchanging and calculating the link-state information need to consume more network bandwidth and node resources. The aim of predicting encounter probability and link-state is to choose an appropriate next-hop forwarding node. Making choice for the next-hop node can control the number of copies which are injected into network and reduce network overhead. But it leads to increasing the potential risk of packet loss rate. How to guarantee success rate of data transmission and reduce network overhead as much as possible is a topic for further researching. Compared with proactive routing protocols, reactive routing protocols can do better in reducing costs. But the latency may be longer. Each has its advantages, so we should make a decision according to the demand in specific environment.

\section{Simulation and Analysis of Routing Protocol}

We adopt ONE (Opportunity Networking Environment) simulator based on discrete event developed by network lab of the Helsinki University to complete the opportunistic networks routing protocol simulation analysis.

\subsection{Basic Scenario Settings}

According the characteristics of performance comparison between WLAN and MANET [39]. We consider several parameters to be set. Experiment simulates pedestrians with smart phones walking in the real city. We attempt to simulate the randomness of the network topology change, discontinuity of communication link, information transmission delay. In general, we set experimental scenarios after repeated experiments according to the coverage of wireless mobile self-organizing network, operating range, network mode etc. We have simulated MessageFerry (MF), Infostation (IF), DirectDelivery (DD), FirstContact (FC), Prophet (PRO), MaxProp (MPRO), Epidemic (EP), Spray and Wait (SNW). The specific parameter settings of simulation is shown in table 2 (The message copy number (nrofCopies) of SNW routing algorithm is settled to 6): 
Table 2. Simulation Parameter

\begin{tabular}{ccc}
\hline Category & Parameter & Value \\
\hline \multirow{3}{*}{ Scenario } & Simulation time & $43200 \mathrm{~s}(12 \mathrm{~h})$ \\
& Area & $4500 \mathrm{mX} 3400 \mathrm{~m}$ \\
& City & Helsinki \\
& Move Speed & $0.5 \mathrm{~m} / \mathrm{s} \sim 1.5 \mathrm{~m} / \mathrm{s}$ \\
Node & Transmit Speed & $250 \mathrm{~KB} / \mathrm{s}$ \\
& Transmit Range & $10 \mathrm{~m}$ \\
& Movement Model & ShortestPathMovement \\
& Buffer Size & $5 \mathrm{M}$ \\
& Number of Node & 100 \\
Packet & Packet Size & $500 \mathrm{k} \sim 1 \mathrm{M}$ \\
& Message TTL & $300 \mathrm{~m}(5 \mathrm{~h})$ \\
& Message Interval & $25 \mathrm{~s} \sim 35 \mathrm{~s}$ \\
\hline
\end{tabular}

\subsection{Analysis of Simulation Results}

\subsubsection{Evaluation Factors of Routing Protocol}

Routing protocol performance has a lot of ways to evaluate. In order to analyze different opportunistic networks routing protocols accurately and comprehensively, we use the following evaluation factors as following:

Delivery ratio: the number of messages generated by the source node in the simulation time and successfully sent to the destination node compared with the number of all messages generated in the network, not including copies produced by different relaying strategies.

Dropped Packets: the number discarded of message when finishing simulation. Packet loss is generally caused by the network nodes congestion or expiry of the message survival.

Latency: the time from source to destination. Actually lowering latency brings about the saving of cache and bandwidth and has better real-time performance.

Routing overhead: the ratio between total numbers of data packet that nodes forwarded in network in a certain time and the number of messages relayed to the destination. The greater routing overhead, the more resources consumed.

\subsubsection{Comprehensive Evaluation of Routing Protocol}

We set the nodes' cache to 5MByte and nodes' number to 100 . Experimental results are shown in Figure 8. Infostation is the simulation under the condition of fixed infrastructure with Infostation routing protocol. MessageFerry is the simulation under the condition of mobile infrastructure with Ferry applied with FC, DD, EP, MPRO, PRO and SAW routing protocol. NonInfrasture is the simulation under the condition of no infrastructure with the above-mentioned 6 routing protocols. According to Figure 8 we can draw the following conclusions:

Infrastructure-based routing protocols perform better than others without infrastructure. Mobile infrastructure-based perform better than fixed infrastructure.

Infostation belongs to fixed infrastructure routing protocol whose network nodes are divided into normal nodes and Infostation base stations. Nodes transmit message to the base stations. The base stations use a wide scope of communication range. That characteristics of high capacity service network nodes can improve the network performance greatly. The performance of Infostation is better than non-infrastructure based routing protocol which can be seen from Figure 8 . 


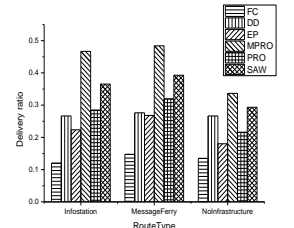

(A)Delivery Ratio of Different Protocols

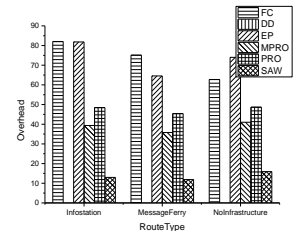

(B)Overhead of Different Protocols

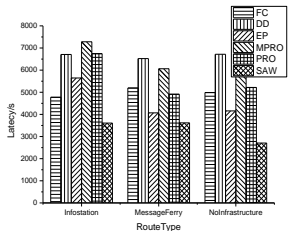

(C) Latency of Different Protocols

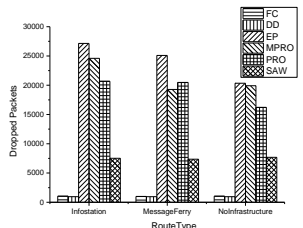

(D)Packets Dropped of Different Protocols

\section{Figure 8. Performance Comparison of Different Routing Protocols for Opportunistic Networks}

MessageFerry belongs to the mobile infrastructure based routing protocol. In opportunistic networks based on MessageFerry, network nodes are divided into two categories: ferry nodes and normal nodes. This article assumes that all normal nodes are equipped with the same radio equipment so that they have the same scope of wireless communication. Ferry nodes are stationary or mobile stationary network devices. They are distributed in a setting area and move randomly in this area. Ferry node is a kind of special node in opportunistic network with the characteristics of high speed, large cache capacity and abundant energy. The main function of ferry node is to forward the message and provide communication services between nodes. Assuming that every ferry node makes random walk in an area with a certain speed. As it can be seen from the experimental results that the ferry has high packet delivery rate and low delay compared with routing protocols without infrastructure. At the same time, ferry is better than the Infostation routing protocol because ferry can move in the network environment, increasing more meeting opportunities. There is no significant difference between overhead and packet loss.

FirstContact and DirectDelivery both belong to the single copy routing protocol. Their network overhead is quite low. The cost of DirectDelivery is zero because it transmits directly to the destination without any overhead of relay. But transfer rate is rather low and delay is much longer without using any prior knowledge.

Epidemic belongings to multi-copy flooding routing protocol. It achieves high data transmitting rate under the condition of the network resources permission while leading to a large number of copies remains in the network and wasting a lot of network resources due to large numbers of flooding. The more packet loss and routing overhead, the higher the delay.

Prophet and MaxProp belong to multiple copy routing protocol based on utility. Prophet distributes messages according to the information that nodes meet in history. Compared with epidemic, they reduce a lot of redundant copies, packet loss, delay and routing overhead. They also increase the packet transmission ratio to some extent. Though they both belong to flooding transmission mode, MaxProp improves and optimizes the Prophet. It manages network copies through the ACK mechanism which can effectively integrate network resources and reduce the number of packet loss and delay. MaxProp is based on the historical knowledge and the strategy of shortest path which can effectively improve the packet transmission ratio, lower routing overhead and latency.

SprayandWait belongs to limited copies and forwarding routing protocol. They copy and distribute limited copies in the message transferring process which can effectively make a balance between the network traffic and resource allocation in aspect of improving the possibility of message transmission. At the same time, limited message copies result in less overhead of network resources, less message transmission delay and packet loss. 
SprayandFocus is based on SprayandWait implementing the high data transmitting rate compared with SprayandWait. The overhead ratio of data transmission and delay have significantly reduced due to focus phase which can further transmit messages.

\subsubsection{Performance Evaluation under Different Node Density and Cache}

\section{A. Routing Protocols Performance under Different Node Density}

According to the parameters aforementioned in Table 2, we change the number of nodes to $10,20,40,100,200,400$ respectively. With the increment of node density under a certain bandwidth and speed, nodes can get higher chances to meet each other and each message can get more chances to be forwarded resulting in the increasing of data delivery rate. Meanwhile the total cache in entire network increases too. Messages can get more chance of being stored during forwarding process. In return, the probability of messages discarded will decline which also enhances the reliability of data transmission.

Figure 9 is the simulation results of six typical non-infrastructure based routing protocols: FirstContact (FC), DirectDelivery(DD), Epidemic (EP), MaxProp(MPRO), PROPHET(PRO) and SprayandWait (SNF) under the condition of six node densities. Figure 9(A) shows that: the increasing node density results in the increasing communication opportunity between nodes. The opportunity and proportion of messages successfully sent to the destination node will be increased greatly. The probability of messages discarded decreases and so does the number of packet loss (shown in Figure 9(D)). Figure 9(B) shows the influence of node density on routing overhead. For that messages can get more forwarding opportunities, hop count from the source to destination increases at the same time. Epidemic will flood more message copies in the time when connection is available. Prophet will collect and calculate more information about meeting information to determine the forwarding path. Both of them will increase the network resource consumption and systematic cost during network running time. Due to the "storage - carry -forward" mechanism, the quantity stored and buffering time carried by intermediate node will increase too. Leading to the increase of network average delay which can be shown in Figure 9(C).

Figure 10 shows the results of MessageFerry infrastructure based routing protocol under different node density. From the figures, you can see that the overall routing performance under infrastructure is better than that without infrastructure. Different node densities have the same tendency. It also shows that the performance improvement should focus on the routing protocol itself. When node density increases gradually, the message delivery rate increases and delay gradually declines. Because of too many nodes involving in transmission, leading to the increase of routing overhead and the packet loss. However, on the whole, the overall routing performance has improved with the increase of network node density.

Concluded above, we can easily find out that the density of nodes distribution directly affects the performance of routing protocol so as to the overall performance of opportunistic networks. From the simulation results, we can see that no matter what kind of routing protocol utilized under the condition of sparse node distribution, the entire network shows the characteristic of low data transfer rate and high packet loss. There are some other factors that make the entire network data transmission performance difficult to guarantee, such as randomness of node movement, uncertainty of messages communication, dynamic change of network conditions, frequent changes of network topology, interrupt of communication link, relatively longer transmission delay in actual condition. 


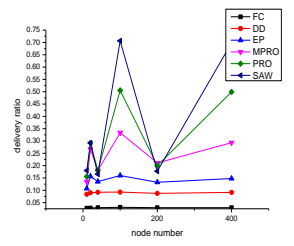

(A)Node Density vs Delivery Ratio

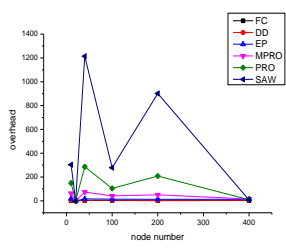

(B)Node Density vs Overhead

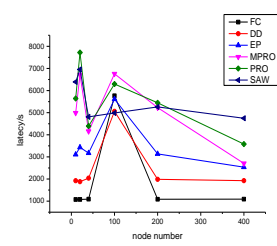

(C)Node Density vs Latency

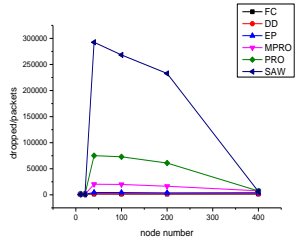

(D)Node Density vs Packet Loss

Figure 9. Node Density of Non-infrastructure Based vs Performance of Routing Protocols under Opportunistic Networks

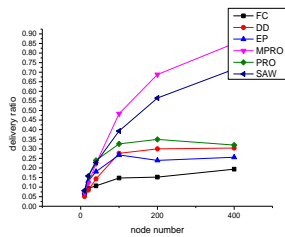

(A)Node Density vs Delivery Ratio

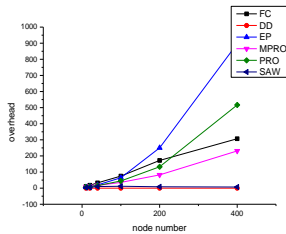

(B)Node Density vs Overhead

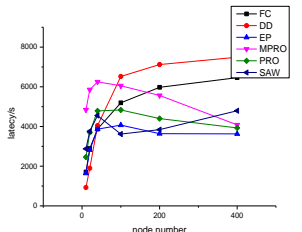

(C)Node Density vs Latency

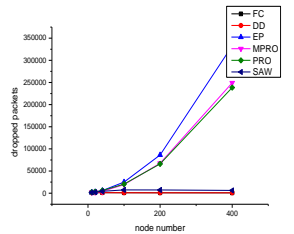

(D)Node Density vs Packet Loss

Figure 10. Node Density of Infrastructure Based vs Performance of Routing Protocols under Opportunistic Networks

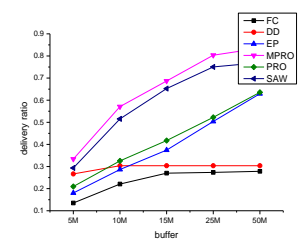

(A)Buffer Size vs Delivery Ratio

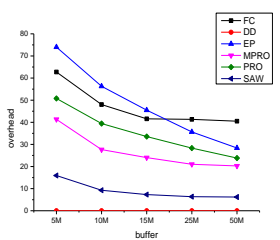

(B)Buffer Size vs Ratio

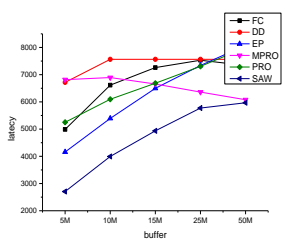

(C)Buffer Size vs Latency

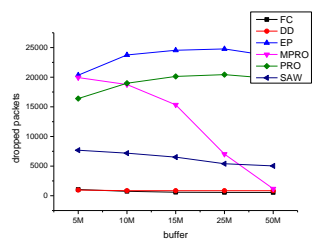

(D)Buffer Size vs Packet Loss

Figure 11. Buffer Size of Non-infrastructure Based vs Performance of Routing Protocols under Opportunistic Networks

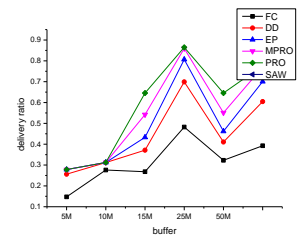

(A)Buffer Size vs Delivery Ratio

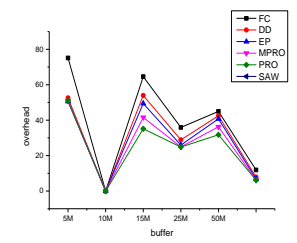

(B)Buffer Size vs Ratio

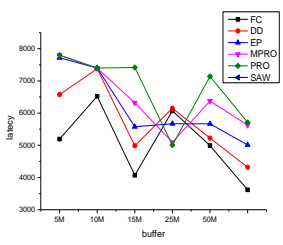

(C)Buffer Size vs Latency

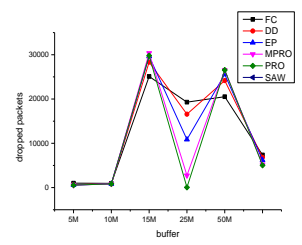

(D)Buffer Size vs Packet Loss

Figure 12. Buffer Size of Infrastructure Based vs Performance of Routing Protocols under Opportunistic Networks

\section{B. Routing Protocols Performance under Different Cache}

Based on the settings above-mentioned, we set the buffer size to $5 \mathrm{M}, 10 \mathrm{M}, 15 \mathrm{M}, 25 \mathrm{M}$, and 50M. With the increment of buffer size under a certain bandwidth and speed, more spaces can be used to cache message so that each message can get a better opportunity to be transferred. Meanwhile the "total cache" in entire network will increase too. Messages can get more chance of 'storage-carry-forward' during forwarding process. Consequently, the probability of messages discarded will decline as well as enhance the reliability of data transmission. 
Simulation results of six typical protocols (FC, DD, EP, MPRO, PRO, and SAW) under 5 caches can be seen in Figure 11 and 12. Figure 11(A) shows message delivery ratio under different buffer size. With the increase of buffer size, messages can be cached until the destination. But the longer cache time, the more packets dropped which can be seen in Figure 11(D). Figure 11(C) shows that the latency increase as the buffer size increase. Figure 11(B) shows that the increasing message cached results in more overhead.

Figure 12 is the simulation results under the protocol of MessageFerry. It shows the same tendency as above-mentioned analysis.

Based on the analysis aforesaid, we find that the cache not only affects the performance of the routing protocols, but also affects the overall performance. The experiments above show that the performance is relatively low when the buffer size is small. But with the increasing in the buffer size, the success delivery rate is also increased. The delay increases for packets cache too long. The overhead is the same as the delay. The number of packets dropped remain unchanged (Figure 12 is more obvious).

4) Evaluation of the applicability of routing protocol

Simulation results show that different routing protocols have different performances. But in the view of priori knowledge utilization, the more using of network history information the better performances are [29] (shown in figure 13).

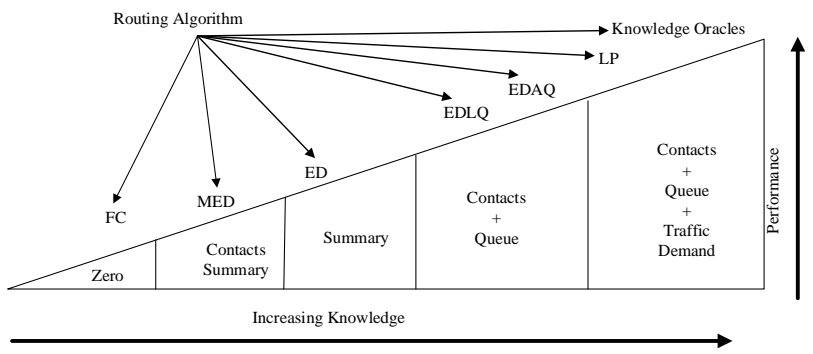

Figure 13. Performance vs. Knowledge Trade-off

The $\mathrm{x}$-axis shows the amount of knowledge (increasing in the positive direction). The $y$-axis shows the expected performance that can be achieved using a certain amount of knowledge. Figure 13 shows that more knowledge is required to attain better performance.

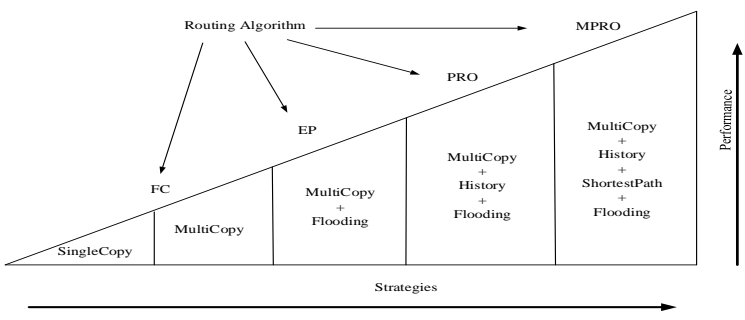

Figure 14. Strategies vs Performance Trade-off

It can be seen from Figure 14 that routing protocols with multi-copy flooding strategy acquire a relatively high packet delivery rate and transmission reliability. But information exchanges so frequently that the buffer between nodes always exceeds its capacity. It consumes most of the network resources. Controlled flooding only forwards limited 
number of packets during transmission which keeps a low overhead, but the successful delivery rate keeps low.

The infrastructure deployed in networks contributes to the total delivery rate. Mobile infrastructure achieves better delivery rate and less overhead compared to fixed infrastructure. But the infrastructure needs more funding which is a key factor when deploying the network.

Regardless of flooding-based, forwarding or hybrid routing mechanism, current available opportunistic network routing protocols are to play their effectiveness in certain scenes. But the condition of opportunistic networks are rather complicated. A variety of protocols are to save storage and energy consumption, the other reduce the transmission delay, or increase the data transfer rate. It's difficult to take all the circumstances into account. No effective, unified way to achieve energy and routing cache capacity constraints, and to improve overall network performance and other factors so far.

\section{Conclusions}

Opportunistic networks are emerging system that's getting growing interest in networking research community. Opportunistic networks lay different research challenges on different layers of a protocol stack. In this paper we provide a quick overview of the state-of-the-art work in providing solutions to various issues in an opportunistic network. This work is aimed to serve as an introductory material to people who is interested in pursuing research in this area.

Typical applications which are characterized by the opportunistic network make the opportunistic network routing problems more complex and challenging. Different applications' needs and performance requirements of routing strategy are the main basis for us to choose the appropriate protocol. Using different routing protocols in different application scenarios, the key is to find a balance overall performance based on requirements between the transmission delay, data transfer rates and network consumption and other constraints in order to maximize the opportunistic network performance. This paper summarizes the basic theoretical knowledge of opportunistic network and its routing protocol. It simulates and analyzes the opportunistic network routing protocols. Experimental results show that different routing protocols using different policies for different application requirements. Particularly, sparse opportunistic network routing will face greater challenges. How to transfer message reliably in a sparse network and how to connect to cloud services would be the focus of our future work.

\section{Acknowledgements}

This work is supported by the National Natural Science Foundation of China (no. 61103248). The authors are grateful for the anonymous reviewers who made constructive comments and improvements.

\section{References}

[1] G. Mohandas, S. Silas and S. Sam, "Survey on Routing Protocols on Mobile Adhoc Networks", International Multi-conference on Automation, Computing, Communication, Control and Compressed Sensing (IMAC4s), (2013) March; Kerala, India

[2] S. Pal, D. Bhattacharyya, G. S. Tomar, Tai-hoon Kim, "Wireless Sensor Networks and Its Routing Protocols: a Comparative Study", 2010 International Conference on Computational Intelligence and Communication Networks (CICN), (2010) December; Nanning, China

[3] J. Kim and S. Kim, "A Study of Multi-Hop Wireless Networks Model for Smart Vehicle Adaptive Traffic", International Journal of Multimedia and Ubiquitous Engineering , vol.8, no.6, (2013).

[4] S.-K. Lee, J.-G. Koh and C.-R. Jung, "An Energy-Efficient QoS-aware Routing Algorithm for Wireless Multimedia Sensor Networks", International Journal of Multimedia and Ubiquitous Engineering, vol.9, no.2, (2014). 
[5] R. Biswas, D. Bhattacharyya, S.K. Bandyopadhyay, "Rapidly Deployable Ad-hoc Network (RADAN) Architecture for Crisis Handling -- a Generic Design", 2012 Fourth International Conference on Computational Intelligence and Communication Networks (cicn), (2012) November; Mathura, India

[6] L. Pelusi, A. Passarella and M. Conti, "Opportunistic Networking: Data Forwarding in Disconnected Mobile Ad Hoc Networks", Communications Magazine, vol. 44, no. 11, (2006)

[7] M. J. Khabbaz, C. M. Assi and W. F. Fawaz, "Disruption-tolerant Networking: a Comprehensive Survey on Recent Developments and Persisting Challenge", Communications Surveys \& Tutorials, vol. 14, no. 2, (2012).

[8] C. Huang, K. Lan and C. Tsai, "A Survey of Opportunistic Networks", International Conference on Advanced Information Networking and Applications Workshops, (2008) March; Okinawa, Japan

[9] P. Juang, Oki H and Y. Wang, "Energy-Efficient computing for wildlife tracking: Design tradeoffs and early experiences with ZebraNet", International Conference on Architectural Support for Programming Languages and Operating Systems, (2002) Oct; San Jose, CA

[10] T. Small and Z. Haas, "The shared wireless infostation model: A new ad hoc networking paradigm (or where there is a whale, there is a way)", International Symposlum on Mobile Ad Hoc Networking and Computing, (2013) June; New York, USA.

[11] M. Musolesi, Dartmouth Coll and Dartmouth, "Writing on the Clean Slate: Implementing a Sociallyaware Protocol in Haggle", International Symposium on a World of Wireless, Mobile and Multimedia Networks, (2008) June; CA, USA.

[12] A. Pentland, R. Fletcher, and A. Hasson, "Daknet: Rethinking Connectivity in Developing Nations", Computer, vol. 37 , no. 1 , (2004).

[13] J. Lu and X. Wang, "Research on the Evaluation of Member Performance in Cartel", Power and Energy Engineering Conference (APPEEC), (2011) June; Wuhan, China

[14] L. Tang, Q. Zheng and J. Liu, "Smart: a Selective Controlled-flooding Routing for Delay Tolerant Networks", Broadband Communications, Networks and Systems, (2007) May; Raleigh, USA.

[15] J. Burgess, B. Gallagher, D. Jensen, B. Levine, "MaxProp: Routing for Vehicle-Based Disruption-Tolerant Networks", INFOCOM 2006 25th IEEE International Conference on Computer Communications, Joint Conference of the IEEE Computer and Communications Societies, (2006) April; Barcelona, Spain.

[16] A. Lindgren, A. Doria, and O. Schelén, "Probabilistic routing in intermittently connected networks", Proc. SAPIR Workshop, (2004) Aug; Shanghai, China

[17] A. Balasubramanian and B. Levine and A. Venkataramani "Replication Routing in DTNs: a Resource Allocation Approach", IEEE/ACM Transactions on Networking, vol. 18, no. 2, (2010).

[18] E. Jones, L. Li and J. Schmidtke, "Practical Routing in Delay-tolerant Networks", IEEE Transactions on Mobile Computing, vol. 6, no. 8, (2007).

[19] B. Poonguzharselvi and V. Vetriselvi, "Survey on Routing Algorithms in Opportunistic Networks", Computer Communication and Informatics (ICCCI), (2013) April; Coimbatore, India.

[20] D. Goodman, J. Borras and B. Mandayam, "Infostations: a New System Model for Data and Messaging Services", Vehicular Technology Conference, (1997) May; Phoenix, USA.

[21] V. Becker, "Epidemic routing for partially connected ad hoc networks", Technique Report, Department of Computer Science, Duke University, vol. 20, no. 6, (2000).

[22] T. Spyropoulos, K. Psounis and C. Raghavendra, "Spray and wait: An efficient routing scheme for intermittently connected mobile networks", ACM SIGCOMM Workshop on Delay-Tolerant Networking, (2005) May; New York, USA.

[23] J. Leguay, T. Friedman and V. Conan, "DTN routing in a mobility pattern space", ACM SIGCOMM Workshop on Delay-Tolerant Networking, (2005) May; New York, USA.

[24] A. Lindgren, A. Doria and O. Schelén, "Probabilistic routing in intermittently connected networks", ACM SIGMOBILE Mobile Computing and Communications Review, vol. 7, no. 3, (2003)

[25] E. Jones, L. Li, and P. Ward, "Practical routing in delay-tolerant networks", Proc. ACM CHANTS, (2003) Aug; New York, USA.

[26] M. Chuah and P. Yang, "A Message Ferrying Scheme with Differentiated Services", Military Communications Conference, (2005); Atlantic City, USA.

[27] R. Shah, S. Roy and S. Jain, "Data Mules: Modeling a Three-tier Architecture for Sparse Sensor Networks", Sensor Network Protocols and Applications, (2003) Feb; Oregon, USA.

[28] M. Musolesi, S. Hailes and C. Mascolo, "Adaptive Routing for Intermittently Connected Mobile Ad Hoc Networks", World of Wireless Mobile and Multimedia Networks, (2005) Aug; Taormina-Giardini Naxos, Italy.

[29] S. Jain, R. Fall and K. Patra, "Routing in a delay tolerant network", Proceedings of the ACM SIGCOMM 2004 Conference on Applications, Technologies, Architectures, and Protocols for Computer Communication, (2004) Aug; Oregon, USA.

[30] M. Grossglauser and D. Tse, "Mobility Increases the Capacity of Ad Hoc Wireless Networks", IEEE/ACM Transactions on Networking, vol. 10, no. 4, (2002). 
[31] K. Harras, K. Almeroth and E. Belding-royer, "Delay Tolerant Mobile Networks (DTMNs): Controlled Flooding in Sparse Mobile Networks", Proceedings of the 4th International Conference on Networking, (2005) Jan; Berlin, Germany.

[32] T. Spyropoulos, K. Psounis and C. Raghavendra, "Spray and Focus: Efficient Mobility-assisted Routing for Heterogeneous and Correlated Mobility", Pervasive Computing and Communications Workshops, (2007) June; White Plains,USA.

[33] Y. Wang, S. Jain and M. Martonosi, "A Message Ferrying Scheme with Differentiated Services", Proc. of the 2005 ACM SIGCOMM Workshop on Delay-Tolerant Networking, (2005) July; New York, USA.

[34] J. Widmer and J. Le Boudec, "Network coding for efficient communication in extreme networks", Proceedings of the 2005 ACM SIGCOMM workshop on Delay-tolerant networking, (2005) Aug; New York, USA.

[35] B. Park, S. Lee and H. Latchman, "A Fast Neighbor Discovery and Dad Scheme for Fast Handover in Mobile Ipv6 Networks", (2006), International Conference on Networking, International Conference on Systems and International Conference on Mobile Communications and Learning Technologies, (2006) April; Morne, Mauritius.

[36] M. Grossglauser and D. Tse, "Mobility increases the capacity of ad-hoc wireless networks", INFOCOM 2001, "Twentieth Annual Joint Conference of the IEEE Computer and Communications Societies", Proceedings, IEEE, (2001) Aug; Anchorage,USA

[37] M. Mitzenmacher, "Digital fountains: A survey and look forward", In Information Theory Workshop, (2004) October; TX, USA

[38] L. Chen, C. Yu and T. Sun, "A hybrid routing approach for opportunistic networks", Proceedings of the 2006 SIGCOMM workshop on Challenged networks, (2006) Aug; New York, USA.

[39] G. Amvame-nze, F.E. De deus, R. Lambert , C. Jacy Barenco Abbas and L. J. García Villalba, "Performance of Wlan and Manet Networks for New Auto-configured Mobile Ip Agents", International Telecommunications Symposium, (2006) September; Fortaleza-Ce, Brazil.

\section{Authors}

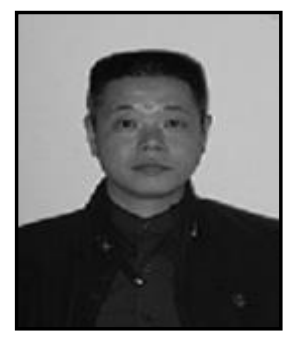

Wentao Wang, he received M.S. degrees in Computer Application Technology from South Central University for Nationalities, Wuhan, China, in 2001, and received the Ph.D. degree in Control science and Engineering from Huazhong University of Science and Technology in 2010. Also, from 2002 to 2003, he worked as a visiting scholar at department of Computer Engineering, Chonbuk National University, South Korea. His current research interests include mobile and sensor networks, image processing, and computational intelligence.

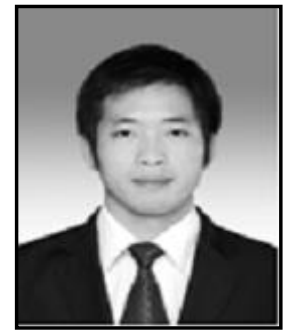

Feng Guo, he received the Bachelor of Engineering degree in Computer Science and Technology from South-Central University for Nationalities, Wuhan, China, in 2012. He is a graduate of Computer application technology in College of Computer Science of South-Central University for Nationalities. His research interests include Wireless Sensor Network, Opportunistic Network, Software Defined Network.

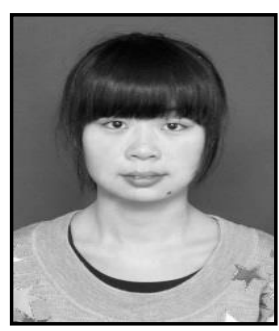

Fang Zheng, she received bachelor degree in electronic and information engineering from Huazhong University of Science and Technology Wuchang Branch in 2012 and now studying for her master Degree of computer application technology in SouthCentral University for Nationalities. Her research interesting includes computer networks. 


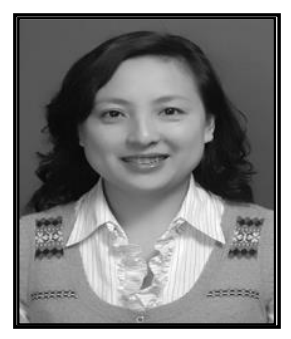

Wan Tang, she received the B.S. and M.S. degrees in Computer Application Technology from South Central University for Nationalities, Wuhan, China, in 1995 and 2001, respectively, and received the Ph.D. degree in Communication and Information System from Wuhan University, China in 2009. She is currently an associate professor in the School of Computer Science of South-Central University for Nationalities. Also, from 2001 to 2002, she worked as a visiting scholar at department of Computer Engineering, Chonbuk National University, South Korea. Furthermore, from 2012 to 2013, she worked as a visiting scholar at department of Computer Science and Engineering, SUNY at Buffalo, USA. Her research interests include protocols for optical/wireless communication networks, network security, and computational intelligence.

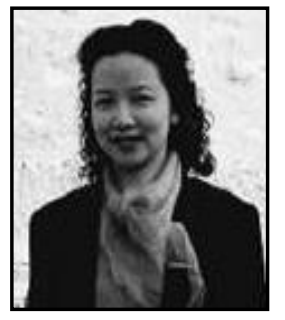

Jiangqing Wang, she received the B.S. and M.S. degrees in Artificial Intelligence from Wuhan University, China, in 1986 and 1989, respectively; and Ph.D. degree in intelligent computation from Wuhan University, China, in 2007. She was a visiting professor of University of Wisconsin-La Crosse and Chonbuk National University. She is currently a Professor in College of Computer Science of South-Central University for Nationalities. She has published over 40 papers in international journals and conferences in the areas of artificial intelligence and intelligent computation. Her current research interests are in the areas of character recognition, intelligent computation and optimization. The research activities have been supported by the Natural Science Foundation of China, Natural Science Foundation of State Ethnic Affairs Commission and Natural Science Foundation of Hubei province. Dr Wang has been actively involved in around 20 international conferences, serving as Session Chair and a reviewer for numerous referred journals and many international conferences. 